\title{
AwareMirror: A Personalized Display Using a Mirror
}

\author{
Kaori Fujinami, Fahim Kawsar, and Tatsuo Nakajima \\ Department of Computer Science, \\ Waseda University, Tokyo, Japan \\ \{fujinami, fahim, tatsuo\}@dcl.info.waseda.ac.jp
}

\begin{abstract}
In this paper, we propose a personalized display, "AwareMirror: an augmented mirror". AwareMirror presents information relevant to a person in front of it by super-imposing his/her image. A toothbrush has been chosen as an identification tool while proximity sensors have been utilized to detect a person's position (in front of the mirror). Also, three types of information that can affect a user's decision have been selected. The mirror has been constructed using an acrylic magic mirror board and ordinal computer monitor. The acrylic board has been attached in front of the monitor, and only bright color from the display can penetrate the board. As a result of preliminary evaluation, we found that the mirror is useful to offer information in an unobtrusive manner while preserving its metaphor.
\end{abstract}

\section{Introduction}

Our daily lives and environments are full of computational devices and a heavy amount of information due to the advancement of technologies. Not only traditional computers, but also small palm sized devices like cellular phones and personal digital assistants (PDA) have network connectivity and offer various information like weather forecasting, today's schedule, etc. This allows people to acquire information anytime anywhere. Also, a new variation of traditional displays like ambient displays[15] are installed into our daily living for showing information to a user. However, if these devices do not provide information in an appropriate way, i.e. considering proper timing, location, identity, and intuitiveness, then computation devices cannot offer comfortable services, because people feel inconvenient to their daily living, and the inconvenience makes them confused how to use the technologies. This means that a user requires to have some efforts to acquire useful information. Offering appropriate information in respective situations is a major research topic in ubiquitous/pervasive computing environments, and this is known as context-awareness.

To extract contextual information of a person, we propose to use a sensor augmented everyday artefact named "Sentient Artefact" [3]. An everyday artefact has a primary role and functionality(-ies). A system retrieves its user's context from the state of these artefacts, and decides on proper contextual responses. A 
stationary daily object like a kitchen sink or a mirror stand can offer a user's location. Moreover, the state of personal belongings such as a purse or toothbrush can be utilized to identify its user. Therefore, we prefer to extract the state-ofuse context by augmenting everyday artefacts with various sensors. Also, we expect to reduce the uncertainty and availability of higher abstract information by integrating various sentient artefacts.

In this paper, we propose a personalized display, AwareMirror, where we have augmented a traditional mirror with some value added services. Using the mirror as a display while preserving its metaphor provides a user with a feeling of seamless augmentation. The contributions of this paper are that 1) it provides a notion of adding values to our daily objects while preserving its metaphor, 2 ) it provides a novel displaying technology using a magic mirror, and 3) it allows to change the mode to show detailed information through a user's explicit interaction.

The structure of the paper is as follows: In Section 2, we introduce a notion of mirror augmentation with cyber world. In Section 3, we examine existing work on context-awareness in terms of utilizing a mirror as an interface, and presenting information in a natural way. We describe the design of the mirror in Section 4, and the prototype implementation is shown in Section 5. Section 6 presents the evaluation regarding the selection of display contents and person identification technologies that are gathered by surveying users. An primary usability testing is also shown. In Section 7, we discuss the issues of the current design and implementation that we have found during the development and evaluation processes. Finally, in Section 8 , we conclude the paper and show future directions.

\section{A Mirror and Its Augmentation with Cyber World}

A mirror has been utilized by people since ancient days. It reflects physical objects appearing in front of it. Often we become inquisitive about our physical appearance by looking at a mirror. Moreover, while using a mirror we can also see and comprehend what is happening in the backgrounds, e.g. someone is passing, a kettle is boiling, etc. This reflective nature is essential for a mirror. We usually stay in front of a mirror for a period of time, which suggests the acceptability of presenting information on the surface of the mirror.

We often think about immediate events or something important e.g. schedule of the day, weather forecasting at our today's destination, etc., while performing something else there. We are able to process or comprehend such a variety of information simultaneously while doing another task in front of a mirror, that is usually the main task, e.g. drying hair, brushing teeth, etc., if the information is shown naturally without disturbing the main task (See Fig. 1).

Our hypothesis is that a mirror which displays information representing people's context can be easily accepted by them because of its nature: reflection. We call the mirror AwareMirror. The mirror utilizes people's prior understandings of a traditional mirror and augments its target to reflect both physical and virtual 


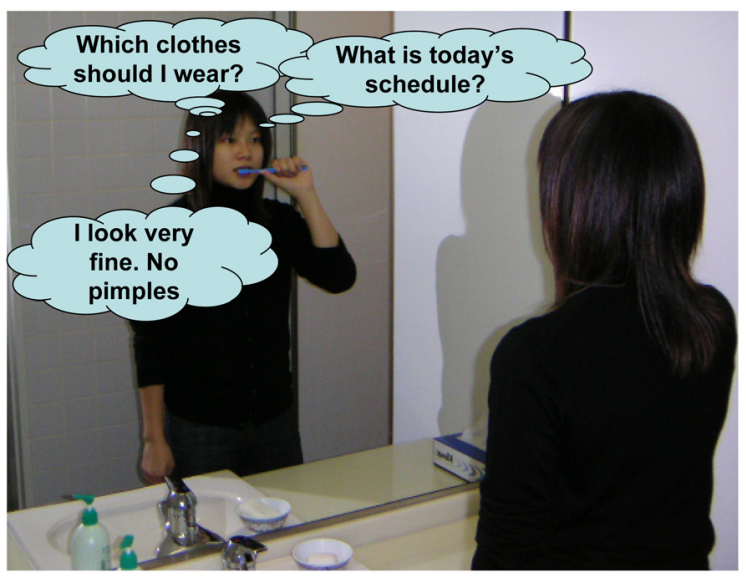

Fig. 1. Multi-tasking in front of a mirror

world. This allows a user to be unconscious of its existence although it provides information relevant to them. The important and difficult design principle is that the mirror should be a traditional one first of all. This means that the functionality as a display should not go beyond one as a reflector. Otherwise it becomes a display with a mirror, not a mirror with a display.

\section{Related Work}

AwareMirror displays information relevant to a person in front of it. The information reflects his/her current or future status, which is often referred to as contextual information or simply context[1]. So, AwareMirror can be considered as a context-aware system.

There is little work regarding the augmentation or using the metaphor of a mirror. Augmented Mirror[7] is an augmented reality system which overlays virtual objects on the mirror. It utilizes a video monitor and a camera to make a mirror and shows an application that a person can see him/her with virtual eye-glasses super-imposed on his/her image. Although it uses a metaphor of a mirror, it merely provides an application of image processing technologies. Next, i-mirror[14] is proposed based on the principle that information services and interactive experiences should be offered by enhancing functions of objects in our life space to provide the ease of use with people. It consists of a video camera, magic mirrors, and a video projector to match the line of sight naturally and proposes applications like historical context displays and virtual make-up systems. Its utilization of a magic mirror to super-impose an image on the mirror is close to ours, however, it heavily depends on video capturing technologies which lead to a feeling of privacy violation. Also, it utilizes a very limited notion of contextual information, i.e. past information of the person in front of it. These two systems just use a metaphor of a mirror, but a user does not use them as an ordinal mirror. 
Regarding to information representation, an ambient display[15] in the context of tangible user interfaces[5] offers an information using human's peripheral awareness capabilities. Our AwareMirror is also an ambient display in a sense because it allows a person to focus on his/her main tasks while offering information in his/her line of sights. The work of Rodenstein [11] can also be categorized into an ambient display. It applies the opposite notion of a mirror and the biggest characteristic of a window, transparency, to overlap information in cyber space, i.e. short-term weather forecast, onto a view of outside. Information visualization with artistic impression like Informative Art [4] and InfoCanvas [13] suggests an "augmented paintings", which can naturally fit into daily living. Their roles are presenting information from the beginning, while AwareMirror has more strict constraints that it should not change its original usage as a mirror while offering additional values, personalized information.

The uniqueness of AwareMirror is using low-level sensing technologies and augmenting the notion of a traditional mirror in a natural way. This is expected to take feelings of obtrusiveness away as well as burden of information retrieval and its understanding. AwareMirror suggests how computing capabilities can infiltrate into daily objects. In the next section we describe the requirements for AwareMirror.

\section{Design of AwareMirror}

\subsection{Requirements}

As described in Section 2, we must preserve the metaphor of the mirror intact. Additionally, the following requirements need to be satisfied for further value addition.

1. Implicit and natural user identification

2. Minimum feelings of privacy violation

3. Types of information and displaying methods suitable for background processing

Information should appear and disappear automatically to keep its natural usage of a mirror and avoid turning it into mere "display device". So, the user should be identified implicitly as well as naturally. This higher level of contextual information should be extracted without using privacy sensitive sensing technologies. A user usually prefers precise information, but he/she denies to use a service if the service uses the vision-based sensing technology because a camera used in the technology causes a feeling to violate privacy. Mostly the location where a mirror is installed is privacy sensitive by considering actions in the location like changing clothes or making up, etc. Therefore, the privacy requirement should be taken into account. We should also consider the characteristics of tasks conducted in front of a mirror to specify required information and displaying methods. AwareMirror is a mirror first of all, which means that a user primarily does his/her main task, e.g. washing face, and he/she might 
feel obstructive if irrelevant information appears during the main task. Also, it should not interrupt the task due to understanding information or hiding his/her own figure by super-imposed information.

These requirements are specific to a mirror and installation place. We believe that the development of artefacts should analyze the requirements that depend on each daily object and its location.

\subsection{Design Issues}

We describe the design issues and approaches of AwareMirror from the several points of view, 1) user detection, 2) display method, 3) contents to show, and 4) modalities of displaying.

Detecting a User: There are several ways to identify a person as follows: 1) face recognition by capturing video images, 2) holding a tag that represents him/her, 3) using biometric information like fingerprint, and 4) acquiring context from everyday artefacts. Biometric-based identification is not appropriate in this case because a user has to put his/her finger explicitly, which might break the first requirement: "implicit and natural identification". The tag-based approach should be rejected due to the same reason. Also, video capturing might provide a feeling of privacy violation.

So, we have utilized the state-of-use of everyday artefacts that are rarely shared with others. Using an everyday artefact's natural functionalities and understanding of people's usage of the artefacts allow an application developer to concentrate on the use of artefacts themselves, rather than the operational environment. Context information can be retrieved from a user's daily activities in a natural way. Such everyday artefacts include a safety razor (an electric shaver), a toothbrush, a comb, etc. We have decided to use a toothbrush for detecting a user because it is gender neutral and utilized by almost everyone.

Three types of information are integrated to detect a user's presence in front of the mirror: 1) the existence of a person in front of a mirror using an infrared range finder, 2) the existence of a toothbrush around the mirror, and 3) the information about the owner of the toothbrush. In Section 6.1, we will discuss the survey of users about alternatives like tag-based, image-based, biometric-based, and artefact-based identifications.

Method of Displaying: We have two options regarding to the method of displaying information on a mirror. One is reflecting on the opposite side of a mirror, e.g. a wall in front of a mirror. The other is penetration behind a mirror. They have both merits and demerits. The reflection method does not require a special mirror. We developed this alternative version first, where a $2 \times 4$ LED-based abstract display that expresses information through the number of lightning is attached on the front wall of an ordinal mirror (see Fig. 2). However, appropriate positioning of the display was required to avoid the LED display being hidden by a user and other persons crossing behind. Adjusting his/her position to an appropriate position leads the person to feel "using a system". Instead, a 


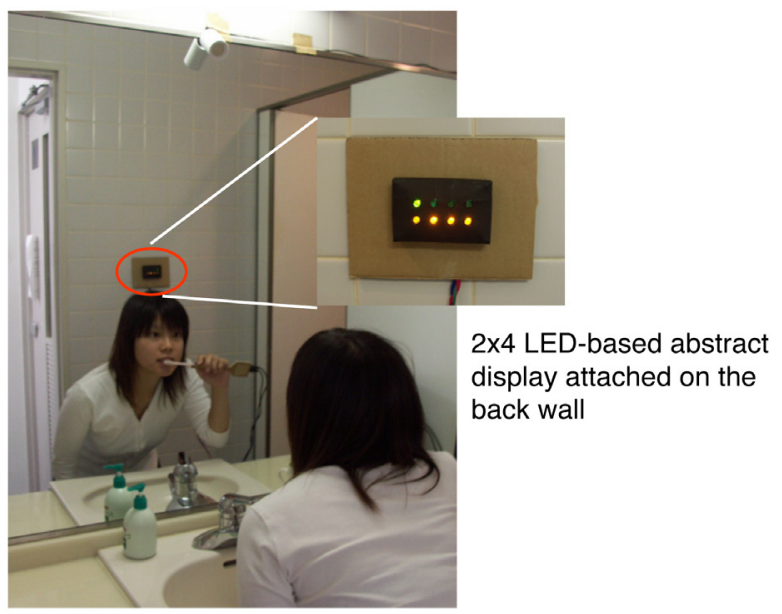

Fig. 2. A mirror with $2 \mathrm{x} 4$ LED-based abstract display

flexible projection system like proposed in Steerable interfaces[9] can change the location of presented information dynamically. But it requires a camera-based positioning system and large mechanical settings which are not suitable for our everyday living spaces. Also, the order of picture and text have to be reversed because of the nature of a mirror. On the other hand, the penetration method requires a special mirror, by which the image and/or text behind the mirror can be seen through. Of course it also reflects physical objects in front of it at the same time. However, we need not take care of a person's position as well as an order of words and direction of pictures. We have selected this option using a "magic mirror". A magic mirror is a translucent panel that reflects things in front of it when the difference of brightness on both sides are large. In other words, things behind the panel can be seen if the brightness difference is small. Therefore, we have utilized this principle to display information.

Types of Information: As introduced in Section 2, we are brushing our teeth, making up, etc., that is normally done in situations where we are considering immediate events or something important over the day to come in the morning. We consider information related to immediate events is useful in such a case. We also often change our decision if someone tells us about weather forecasting, traffic accident, etc. Considering these facts, we have selected the following information to be displayed that can affect our behavior and help decision making.

- Notification and detail information of transportation trouble that the person is going to use by his/her schedule

- Weather forecasting at the destination where he/she will go

- Time to leave his/her home or office for the destination

- Details of the closest event on his/her schedule 
These types of information can remind us of taking something required, e.g. umbrella and documents, and offers us the opportunity to take an alternative route to go to the destination, or rush ourselves. Therefore, we consider that it is appropriate for information presented on a mirror.

Two Modalities of Displaying: Although above listed information can be useful, it might disturb a user's primary task if all information appears on the surface of a mirror. Also, privacy sensitive information can be revealed. Therefore, we have decided to apply two phase interactions with the mirror: implicit and explicit.

In the implicit mode, it plays the role of an ambient display, which shows abstract information on the periphery of a user's line of sight. So, in this mode, people use AwareMirror as an ordinary mirror. Abstract information can be represented using images and colors we are familiar with and therefore easy to understand at a glance. This is also a solution that takes into account of the characteristic of the place, in front of a mirror, since some people often take their glasses off while brushing their teeth and washing their faces. Therefore, we consider that it is useful for them to be just notified of some important events. In the default mode, the mirror does not display anything, so it just looks like a mirror. However, as described above, if a person appears in front of AwareMirror and a toothbrush is utilized, then AwareMirror turns into the implicit mode. It also returns to the default mode on detecting a user's leaving from the place. These transitions occur automatically.

In the explicit mode, however, it presents detailed information, where they explicitly use it as a display rather than a mirror. It shows information in more detail using text information. This mode of interaction might break our design philosophy if we offer detailed information automatically because it could stop a user's current task whether a person wants to get it or not. Therefore, we provide the flexibility to change his/her mode of the usage of the mirror whenever a user wants to change the modes explicitly. On entering into the explicit mode, the role of AwareMirror turns into an information display, not an ordinary mirror. This is caused by the person him/herself explicitly. Hence, we consider that a feeling of disturbance does not come up in his/her mind. We also consider our approach meets the requirements for ambient displays introduced by Mankoff et al[6].: "Easy transition to more in-depth information".

\section{Prototype Implementation}

\subsection{System Architecture}

Figure 3 shows the overall architecture of the AwareMirror system, which consists of four major parts: the physical world information manager, integrated applications, the AwareMirror front end, and the sentient toothbrush.

We consider applications running in a space can share some pieces of information like the location of an object, the state-of-use of the object, its owner(s), etc. Therefore, we have developed a system software called Bazaar to manage 


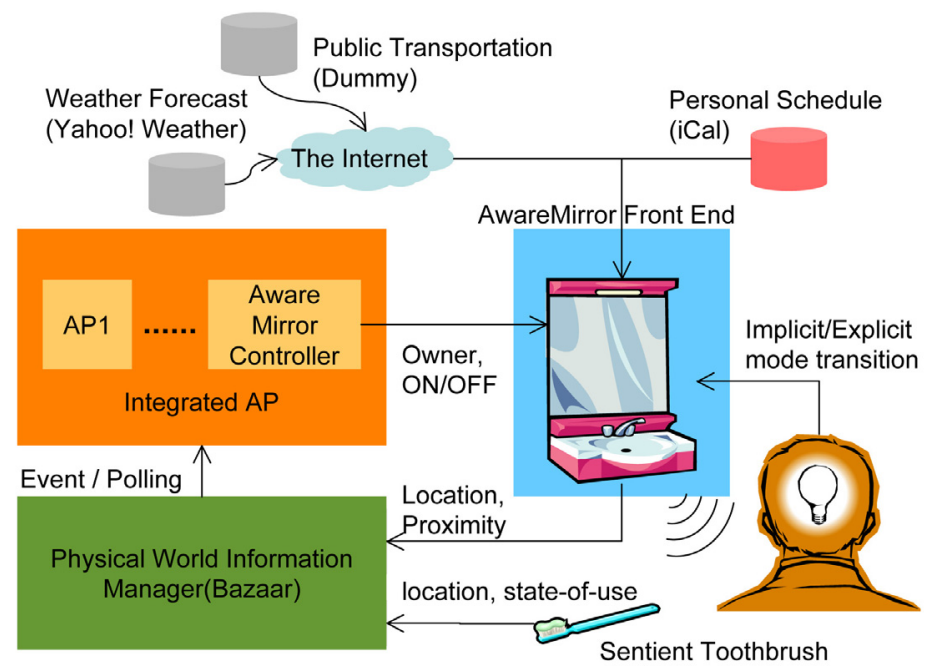

Fig. 3. Overall architecture of AwareMirror

such physical world information separately from each application [2]. Integrated applications that aggregate information from many sentient artefacts are developed on top of Bazaar. The applications also control sentient artefacts by using collected information. In this case, one of integrated applications, the AwareMirror controller, is responsible for notifying the AwareMirror front end of requiring operations, i.e. turning on or off the display. This allows the AwareMirror system to replace various identification technologies, i.e. biometric-based, image-based, etc., according to each application's requirements. When the AwareMirror controller receives two types of events: 1) co-location of a sentient toothbrush used by a person with AwareMirror and 2) presence of him/her in front of the mirror, the AwareMirror controller makes the AwareMirror front end to turn on, and passes the ID of the toothbrush's owner to it.

On the AwareMirror front end, the detection of a user, information rendering, and mode transition by him/her are dealt by five software components to be described in Section 5.3. A personal scheduler, public transportation information service, and weather forecasting service are used as external information sources. Finally, a sensor augmented toothbrush, the sentient toothbrush, detects the beginning and end of brushing.

An ID is assigned to each sentient artefact, which is detected by some recognition systems like a radio frequency identification(RFID) system, and registered into Bazaar. All these components cooperate with each other to offer the implicit and natural interaction between human and augmented artefacts.

\subsection{Hardware Settings of AwareMirror}

Figure 4 shows an overview and assembling scene of AwareMirror. As described in Section 4, information is projected behind the mirror. An acrylic magic mir- 


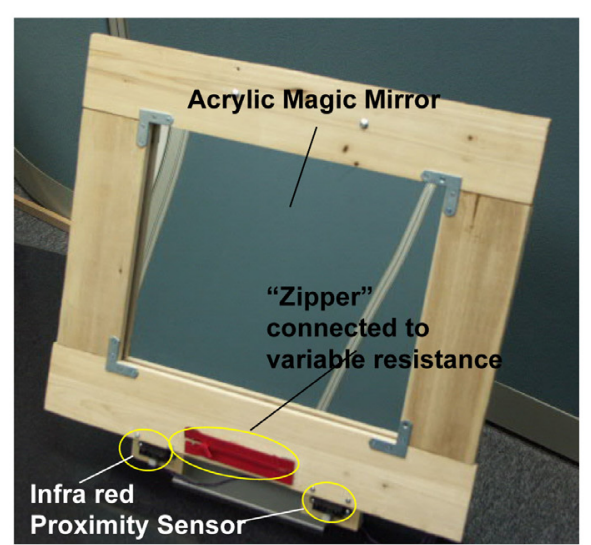

(a) Overview

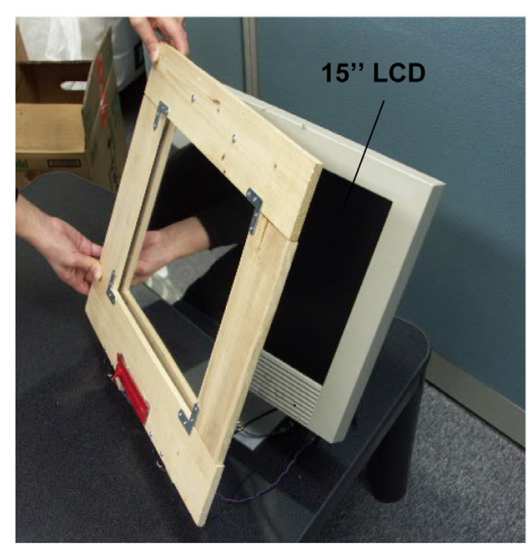

(b) Assembling

Fig. 4. Overview and assembling of AwareMirror

ror is attached on an ordinal liquid crystal display (LCD) and the information is controlled through a Windows-based PC. A "Zipper" metaphor is utilized to achieve transition from the implicit to explicit mode. When a user opens the zipper, the application's operational mode changes to the explicit mode and displays detailed information. The system expects him/her to close the zipper to stop displaying and turn to the default black screen because detailed information may contain highly privacy sensitive information as well as avoid taking mirror's functionalities away. If the user does not open the zipper, the application operates in the implicit mode, and the display is controlled by the application itself based on the control information received from Bazzar. It starts and stops automatically without a user's intervention. One point to note here about the zipper metaphor is that we have used this notion to make the user to feel "Used To" with the system. As we mentioned previously, we want to develop a mirror with some value added services, not a display. We all know how a zipper works and what interaction it needs to offer its service. So the user does not need to learn a new technology. It is natural for us to open a zipper of some containers to get the things inside, in our case, detailed information. Also, the texture and looks of the zipper remind a person of closing, and thus it contributes to avoid keeping relevant information open. A real zipper is connected to a variable resistancebased input device (slider) which is then attached to Phidget Interface Kit[8]. The distance between the mirror and an object in front of it is measured by two Sharp GP2D12[12] infra-red range finders that provide redundant outputs. The sensing range is from $10 \mathrm{~cm}$ to $80 \mathrm{~cm}$, and the field of view is 10 degrees for each. They are also connected to Phidget Interface Kit.

\subsection{Software Components on AwareMirror Front End}

The AwareMirror front end consists of five components: a) Display Controller, b) Interaction Controller, c) Contents Manager, d) Rendering Controller, and e) Human Detector. Display Controller is the heart of the AwareMirror front end, 
which controls an application's control flow. It waits for HTTP-based service requests from Bazzar. Once received the "Turn On Display" request, it constructs information to be displayed through Contents Manager, and the request is delivered to the Rendering Controller to show the contents. On the other hand, if received the "Turn off Display" request, Display Controller closes the display to turn it into the default mode. Also, it monitors a user's interactions through Interaction Controller which handles a user's input to change the mode from implicit to explicit back and forth.

As described in Section 5.1, three types of information source are utilized. We have used the Yahoo ${ }^{T M}$ Japan's weather service and a simulated transportation information service. Currently, iCalendar [10]-based scheduler service "iCal" runs in a Macintosh Powerbook to offer schedule information. We expect this sort of a scheduler service should be provided in personal devices like cell phones or handheld devices in the future. Based on a user's next schedule's location and time, Contents Manager extracts proper weather and transportation information. Such information can vary according to the time of the day. So, Display Controller manages the timing to display based on a user's preference setting.

Rendering Controller renders the information using MacromediaT ${ }^{T M}$ Flash movie because of its aesthetic features and easy to build animation capabilities. An appropriate visual component is selected based on a request from Display Controller. What we need to consider is the color of contents because, due to the characteristics of a magic mirror, dark color is hard to see through the translucent panel.

A user's distance from the two infra-red range finders are transformed into information indicating a user's presence in front of the mirror. So, if a person enters within the sensing range $(10 \mathrm{~cm}$ to $80 \mathrm{~cm})$, an event is transmitted to Bazaar. Also, when a user goes out of the range, the event to notify the absence is delivered.

\subsection{Sentient Toothbrush}

A sentient toothbrush has been augmented with a two-axis accelerometer connected to Phidget Interface Kit. It can detect the start and end of brushing by detecting its vibration. This is achieved by monitoring the change of the acceleration. It reports its change of the state-of-use to Bazaar. Currently, the extraction of the state change is processed on a laptop PC, however, it can be replaced by embedded computers that have wireless connectivities.

\subsection{Using the Mirror}

Figure 5 shows the results of displaying information related to the person in front of it. In Fig. 5-(a), a girl is brushing her teeth while looking at information on the mirror at the periphery of her line of sights. This abstract representation is what is in the implicit mode and initially displayed. In this case, it shows the fact that 1) the weather forecast at her today's destination based on her next schedule is fine with an image of sunshine, 2) the maximum temperature 


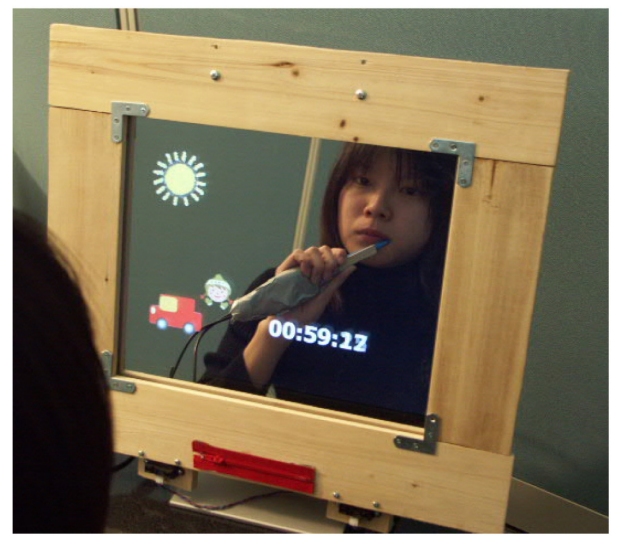

(a) Implicit mode
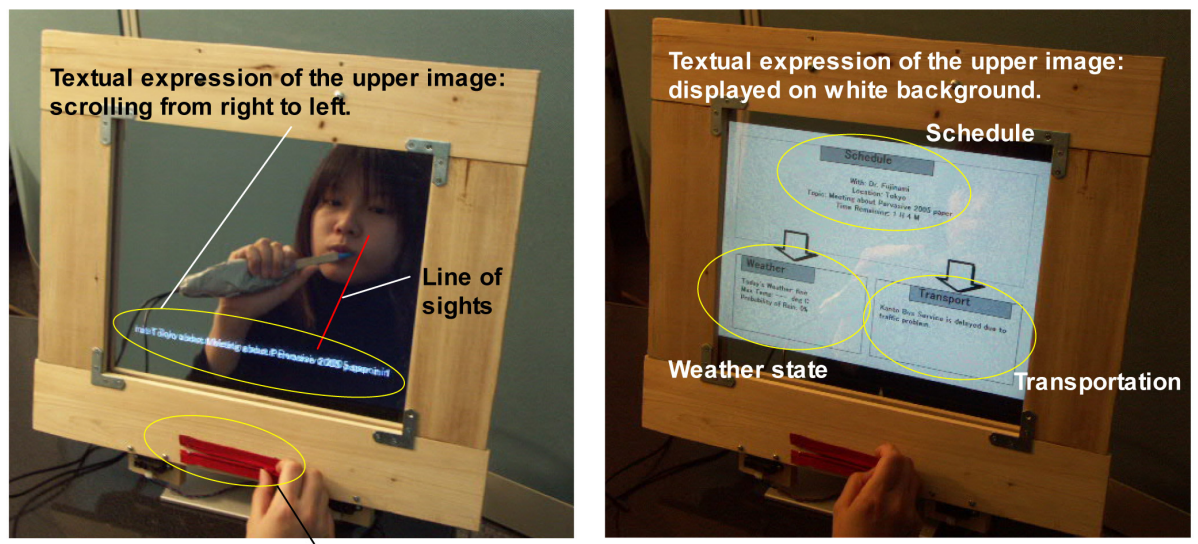

Opening "Zipper" to get detail information by herself.

(b) Explicit mode: Scrolling Text

(c) Explicit mode: black characters with white background

Fig. 5. Usage of AwareMirror, (a) Implicit mode, (b) Explicit mode with scrolling message, and (c) Explicit mode with white background

at her destination is less than 15 degrees Celsius using an image of a girl with gloves and a snow cap, 3) the transportation on the way to her next schedule has some problems indicated with a red car, and 4) time remaining for the next schedule. Her schedule information is utilized as contextual information in the future tense. A screenshot presenting information can be seen in Fig. 6 .

If she wants to know more about the information presented abstractly, she will suspend her current task, i.e. brushing her teeth, and open the "Zipper" to get details by herself (Fig. 5-(b)). By opening the zipper, the following textual information scrolls from the right to left repeatedly:

"The next schedule is a meeing with Mr.Fahim to discuss the Pervasive2005 paper in 59 minutes. Today's weather is fine. Maximum temperature is 9 degree's 


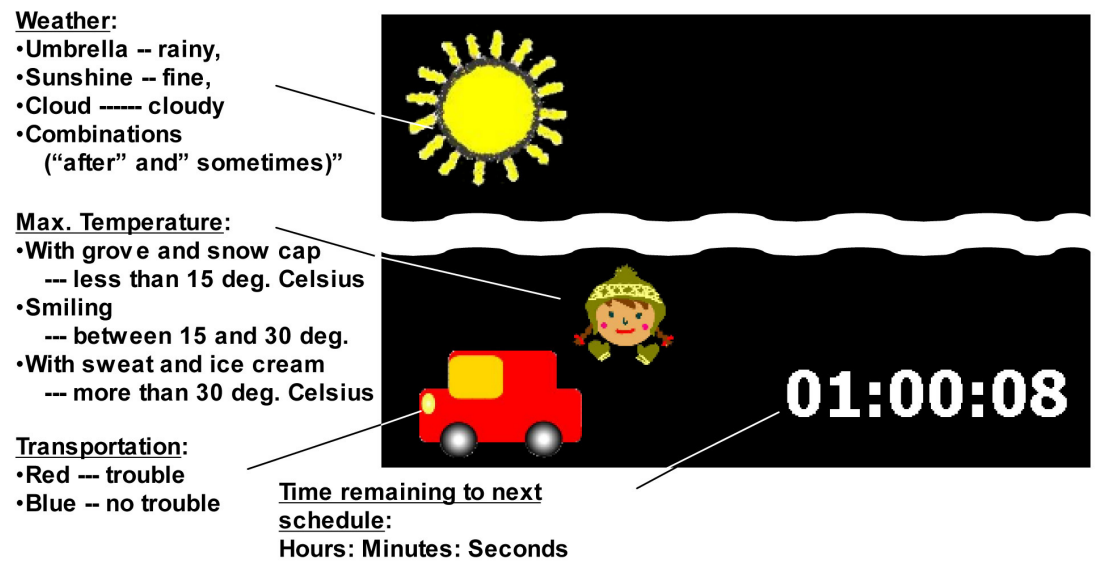

Fig. 6. A screenshot penetrating images from behind

Celsius. The probability of rainfall around your next schedule is 10\%. On your way to the next schedule, Yamanote Line is stopped due to an accident."

Also, we have developed another version of the explicit mode, where textual information is presented in black on the white background (see Fig, 6-(c)). It looks like a document on a traditional computer display, which can lose the functionality of a mirror. However, we have expected that a user might accept this because he/she selects it by him/herself in an explicit way. We will discuss on this preference of users in Section 7.

Thus, our AwareMirror provides contextual information with a person, first seamlessly (implicitly) and then explicitly, while preserving its traditional look and interaction mode as much as possible.

\section{Evaluation}

In this section, we present the results of a user survey regarding the identification methods and display contents. Moreover, we also present the results of user testing. Then, we discuss the requirements and our current design.

\subsection{Preference for Identification Technologies}

We carried out a survey for investigating preferable identification technologies.

Profiles of Testees: The survey was conducted using questionnaires among 50 people. 34 of them are the members of the authors' laboratory including one professor, four Ph.D students, and 29 master course and undergraduate students where 6 of them are female. Approximately 10 of them work on ubiquitous computing projects. The rest of the testees (16 persons) are people who do not 
work in our laboratory including five undergraduate students, five housewives, four company employees, and two artists with ages ranging from 20 to 69 .

Questionnair: We listed four types of identification mechanisms that include: a) carrying a wireless tag whose detection range is approximately $3 \mathrm{~m}$ (tag-based), b) face recognition by video captured images (image-based), c) state of an everyday object that is rarely shared with others (sentient artefact-based), and d) fingerprint or voice recognition (biometric-based). Then, we asked testees to compare them and assign a rank from 1 to 4, where 1 for the least preferred and 4 for the most preferred. Also, a person who chooses c) can identify which artefact is desirable.

The Results: The average score for each method is as follows: biometric-based (2.92), tag-based (2.67), sentient artefact-based (2.27), and finally video-based (2.24). This means that the biometric-based identification was most preferable among the four, while the video-based one was preferred least. The biometricbased approach is preferred because it is considered to provide high accuracy and does not require a user to carry anything. However, the method is claimed to require explicit interaction to identify a user. For the tag-based approach, testees liked to control the timing of displaying information, but they disliked the possibilities to forget or lose the tag.

The merit of the artefact-based one pointed by them is its implicit identification by an artefact that is hardly shared with others like a toothbrush. However, the possibilities of intentional or unintentional use by other persons were pointed by participants who specified a lower rank. Finally, the video-based approach was disliked because of its obtrusive feeling of being watched although they know that it requires no input by a user, and also they know that it can extract human activities like looking at a mirror. The characteristic of a washroom is considered to emphasizes that feeling.

In terms of a daily artefact that is hardly shared with others, a toothbrush was listed by most of the participants. A safety razor or electric shaver, towel, hair brush, and cosmetics were also listed.

\subsection{Preference for Contents}

We also carried out a survey to investigate a user's preference for displayed contents.

Questionnaire: First of all, we made them to understand the notion of AwareMirror. Then, we asked questions about the usefulness of contents to be displayed on the mirror. The testees were allowed to rank the usefulness to 12 imaginary information services, i.e. 1 for "useless, 2 for "weakly useless", 3 for "depends on the current situation", 4 for "fairly useful", and 5 for "very useful". Also, we provided free space with each question for filling further comments. In addition to the information services that are actually utilized (see Section 4.2),we 
provided the following ones which can be categorized as information for entertainment and knowledge: fortune teller, level of beer taste, current news, and email contents.

The Results: Table 1 shows the results of the rating, where the second column represents services that were actually utilized. The third column indicates the average number of the rank while the fourth and the fifth mean the type of information and the level of abstraction, respectively. We have found that the testees preferred decision making information rather than ones that fall in the "knowledge" and "entertainment" category. According to the comments, the reason for the low rating of "knowledge" were that getting the information requires the interaction with the mirror, e.g. selecting and reading a number of e-mail one by one, and the interaction is not desirable at the location where the mirror is installed. On the other hand, a user was not interested in "entertainment" information in front of the mirror. Other types of preferable information they specified include the states of their health, train time table, etc. Remarkably, several testees expected the flexibility of controlling the level of information details. In Table 1, they preferred detailed information as well, however, they were also worried about the exploitation of privacy sensitive information.

Table 1. Preference of contents regarding usefulness

\begin{tabular}{l|c|c|c|c}
\hline Name of information & Used & Average rank & Type & Level \\
\hline \hline Notification of transportation trouble & $\mathrm{x}$ & 4.69 & $\mathrm{DM}$ & $\mathrm{AB}$ \\
\hline Details of transportation trouble & $\mathrm{x}$ & 4.53 & $\mathrm{DM}$ & $\mathrm{DT}$ \\
\hline Weather forecast at destination with text & $\mathrm{x}$ & 4.51 & $\mathrm{DM}$ & $\mathrm{DT}$ \\
\hline Weather forecast at destination with image & $\mathrm{x}$ & 4.23 & $\mathrm{DM}$ & $\mathrm{AB}$ \\
\hline Schedule of the day in details & $\mathrm{x}$ & 3.81 & $\mathrm{DM}$ & $\mathrm{DT}$ \\
\hline Current news & & 3.64 & $\mathrm{KW}$ & $\mathrm{DT}$ \\
\hline Time to departure with image & $\mathrm{x}$ & 3.59 & $\mathrm{DM}$ & $\mathrm{AB}$ \\
\hline Notification of change on the current news & & 3.38 & $\mathrm{KW}$ & $\mathrm{AB}$ \\
\hline Email contents & & 2.98 & $\mathrm{KW}$ & $\mathrm{DT}$ \\
\hline Level of beer taste with image & & 2.37 & $\mathrm{EN}$ & $\mathrm{AB}$ \\
\hline Fortune teller with text & & 2.35 & $\mathrm{EN}$ & $\mathrm{DT}$ \\
\hline Fortune teller with image & & 2.10 & $\mathrm{EN}$ & $\mathrm{AB}$ \\
\hline \multicolumn{2}{c|}{ DM: Decision Making, KW: Knowledge, EN: Entertainment } \\
\hline
\end{tabular}

\subsection{Usability Testing}

We had a test by one family consisting of 3 members (father, mother, son) to get the feedback of the usability.

Methodology: We had two comparative experiments to investigate the effect of the two phase interaction and presentation in the explicit mode. We have 
created AwareMirror without the zipper, where only abstract information is displayed. The testees were provided this version of AwareMirror first and then that with the zipper. In the second experiment, two types of presentations, i.e. scrolling text and black characters on white background (see Fig. 5-(b) and (c)), were compared.

The Results: All three testees seleted AwareMirror with the zipper, which indicates they preferred to have the flexibility of controlling the level of information details. However, they complained about the explicit necessity of closing the zipper by themselves. They pointed out the possibility to forget it since they were often in a hurry in front of a mirror. Also, the non-intuitiveness of the zipper was claimed. Instead, they told that they would like to control by a pushbutton switch like a bell button. The results oppose our initial design described in Section 5.2.

Regarding to the presentation of the explicit mode, they also preferred the version with scrolling text because the white background one gave them obtrusive impression. This suggests that a user regards it as a mirror even if he/she selects to see detailed information. Moreover, there was a request for showing detailed information selectively. Overall, they agreed to the notion of "information perception as secondary task" that AwareMirror is trying to achieve.

\subsection{Summary of Evaluation}

The notion of augmenting a mirror with context sensitive display was well accepted. The presentation of information triggered by using a toothbrush and standing in front of AwareMirror provides testees with feelings of implicit and natural identification that we were intended. However, testees were worried about the possibility of using these artefacts by others which may hamper their privacy. Information that can affect a user's behavior was preferred because, according to testees' comments, he/she can change the next action considering this information. Regarding to a display, abstract information was preferred due to its unobtrusiveness. The two phase interaction was also desirable because of its flexibility of controlling.

\section{Discussions}

In this section, we show some issues in designing and evaluating AwareMirror.

\subsection{Identifying a Person in Front of a Mirror}

We have utilized the state-of-use of a toothbrush for identifying a person. The detection of something in front of a mirror and the co-location of the mirror and toothbrush being used are combined to identify the person in front of the mirror. The sensing technologies used are simple, but this technique may lead to mis-identification. As pointed out in the survey result shown in Section 6.1, an everyday artefact may be used by an unintended person. Also, detecting an artefact status "being used" is difficult. However, these artefacts can be used to 
start showing personalized information on the mirror without a user's explicit interaction. Also, an assumption that it is utilized in a closed reliable group like a family suppress these issues. Moreover, we flexibly select an appropriate way of the identification since the AwareMirror controller encapsulates it.

\subsection{Usability}

Mode Transition by Zipper Metaphor: As described in Section 6.3, most of the users liked the two phase interaction, but they did not want to use the "zipper" for mode transition. AwareMirror turns to the black screen (default mode) when it detects a user's absence in the both implicit and explicit mode. Here, we assume that the zipper remains opened. When a new user comes in front of AwareMirror, it displays abstract information. So, he/she needs to close the zipper first if he/she wants to "open" the zipper to change into the explicit mode, which makes them confused. Moreover, as stated by the testees, they often forgot to close the zipper. We consider the reason for this is the statefulness of the zipper. That is, if a "push button" is utilized, a user does not have to bring it to the normal position. Also, we argue that the non-intuitiveness of the zipper for switching modes comes from its multiple roles. It is utilized not only to extract something from inside (digital value), but also to fasten something at a proper position like a zip-up jacket (analog value).

Presentation of Explicit Mode: According to the user testing, scrolling text was desirable as the presentation in the explicit mode. However, it continuously scrolls from the right to left and takes some time to show all information, while the white background-based approach can offer all information at a glance. In the current version, it takes about 40 seconds to show all information as presented in Section 5.5. This makes it difficult to get only desired information rapidly. The problem may be solved by choosing an appropriate information by touching the information on the mirror directly, but the solution causes another problem described in the next paragraph.

Issues in Showing Detailed Information Selectively: The touch panelbased interaction becomes popular for natural input, however it is inappropriate for applying a mirror. If a user touches on its surface, his/her fingerprint remains. Therefore, it makes the functionality of the mirror useless. Additionally, if buttons are used like function keys, it loses the metaphor of a mirror. We need to provide a natural and suitable interaction method for the mirror.

\subsection{Possible Issues in Large Sized Version of AwareMirror}

The current 15 inches LCD version is small enough to become aware of information displayed. However, in the case of a large mirror, a person might not notice the information if it is displayed on the top and/or bottom corner due to human eye's capability. One option to avoid this is generating sound at the beginning of the display. But the approach requires a user to pay an attention, which might violate our design principle. Therefore, we should consider alternatives while keeping the characteristic pointed in the previous section. 
Also, in the current version, the information cannot be seen by others because the body of the subject covers it. However, in turn, the large sized one like Fig. 1 can have the chance, where more than two persons could use it at a time. To show information to an appropriate user, the location should be identified properly. We consider the current location identification mechanism is inappropriate because it utilizes a wide range RFID tag system which can detect tags in 2 to 3 meters. Therefore, it is difficult to match the detection by the infra-red sensor with the detection by the RFID tag reader.

Regarding to the detection of a person in front of the mirror, it is also difficult since an infra-red range finder usually has the narrow range of detection (in case of GP2D12, it is 10 degrees). For detecting with high accuracy, one solution for this is to attach infra-red sensors densely in front of a faucet while loosely at other places, and this leads to better detection. However, the solution might break aesthetic feeling and might be expensive. We are investigating these issues to deploy AwareMirror in public and private washrooms using a 60 inches plasma display in a practical way.

\section{Conclusions and Future Direction}

In this paper, we have proposed an augmented mirror, AwareMirror. AwareMirror offers abstract and personalized information on a mirror to a person who is in front of the mirror. Our approach preserves the metaphor of a mirror, although the mirror is augmented to offer useful information while a user is washing his/her face or making up. AwareMirror is considered as a new type of ambient displays.

The primary user feedbacks were mostly positive. However, we are working on building and evaluating iteratively through further usability testing. Especially, we need to investigate the usefulness of the explicit mode. Moreover, the size of a display that affects the ease of understanding information, feelings of privacy violation, and sensing technologies should be taken into account in the near future. We have now a 60 inches plasma LCD, and we hope to build a larger augmented mirror to verify the possible issues mentioned above.

\section{Acknowledgments}

We would like to thank Ms. Kanako Okada and Mr. Takeshi Kambe for their constant improvements of the AwareMirror system.

\section{References}

1. A. Dey, G. Abowd, and D. Salber. A Conceptual Framework and a Toolkit for Supporting the Rapid Prototyping of Context-Aware Applications. HUMANCOMPUTER INTERACTION, 16(2-4):97-166, 2001.

2. K. Fujinami and T. Nakajima. Towards System Software for Physical Space Applications. In Proceedings of ACM Symposium on Applied Computing(SAC) 2005, March 2005. (to appear). 
3. K. Fujinami and T. Nakajima. Sentient Artefact: Acquiring Context by using Daily Objects. Technical report, Waseda University, 2005(to appear).

4. L. E. Holmquist and T. Skog. Informative art: Information visualization in everyday environments. In GRAPHITE '03: Proceedings of the 1st international conference on Computer graphics and interactive techniques in Australasia and South East Asia, pages 229-235, 2003.

5. H. Ishii and B. Ullmer. Tangible Bits: Towards Seamless Interfaces between People, Bits and Atoms. In Proceedings of Conference on Human Factors in Computing systems, pages 234-241, 1997.

6. J. Mankoff, A. K.Dey, G. Hsieh, J. Kientz, S. Lederer, and M. Ames. Heuristic evaluation of ambient displays. In Proceedings of the conference on Human factors in computing systems, pages 169-176, 2003.

7. S. Naka, H. Kato, and K. Tachibana. Augmented Mirror: Augmented Reality in the Mirror World. Technical Report TR-MVE 2001-10, The Institute of Electronics, Information, and Communication Engineering(IEICE), June 2001. (in Japanese).

8. Phidgets Inc. Web site. URL: 〈http://www.phidgets.com/〉.

9. G. Pingali, C. Pinhanez, A. Levas, R. Kjeldsen, M. Podlaseck, H. Chen, and N. Sukaviriya. Steerable Interfaces for Pervasive Computing Spaces. In Proceedings of the First IEEE International Conference on Pervasive Computing and Communications(PERCOM'03), pages 315-322, March 2003.

10. RFC2445. Internet Calendaring and Scheduling Core Object Specification (iCalendar). URL: 〈http://www.faqs.org/rfcs/rfc2445.html〉.

11. R. Rodenstein. Employing the Periphery: The Window as Interface. In Extended abstract of International Conference on Human Factors in Computing Systems(CHI'99), pages 204-205, May 1999.

12. Sharp Corporation. Optical System Device. URL: /http://sharp-world.com/ products/device/lineup/table/085.html $\rangle$.

13. J. Stasko, T. Miller, Z. Pousman, C. Plaue, and O. Ullah. Personalized Peripheral Information Awareness through Informative Art. In Proceedings of 6th International Conference on Ubiquitous Computing(Ubicomp2004), pages 18-35, October 2004.

14. K. Ushida, Y. Tanaka, T. Naemura, and H. Harashima. i-mirror: An Interaction / Information Environment Based on a Mirror Metaphor Aiming to Install into Our Life Space. In Proceedings of the 12th International Conference on Artificial Reality and Telexistence (ICAT2002), December 2002.

15. C. Wisneski, H. Ishii, A. Dahley, M. Gorbet, S. Brave, B. Ullmer, and P. Yarin. Ambient Displays: Turning Architectural Space into an Interface between People and Digital Information. In Proceedings of the First International Workshop on Cooperative Buildings (CoBuild'98), pages 22-32, February 1998. 